\title{
Etude des relations entre une racine et les rejets de la souche chez Castanea sativa Mill.
}

\author{
M. AYMARD, J.-J. FREDON \\ Laboratoire de Biologie végétale \\ U.E.R. des Sciences, F 87000 Limoges
}

\begin{abstract}
Résumé
Le rôle de la souche, pour des arbres traités en taillis, est encore imparfaitement connu, surtout en ce qui concerne la répartition des nutriments puisés dans le sol, entre les différents rejets. L'application d'un phytocide (amitrole) sur unc racine d'une cépée de châtaignier provoque le dépérissement de certains rejets et permet ainsi de mettre en évidence les voies de transport utilisées. L'intensité des dégâts provoqués est modulée par l'application de doses plus ou moins importantes de traceur. Deux séries d'expériences ont été réalisées, l'une au printemps, l'autre en automne sur des cépées de 1 et 4 ans. Lorsque l'apport de phytocide est faible, seuls les rejets situés à proximité de la racine donneuse sont atteints. Dans le cas d'un apport plus important, les dégâts peuvent se manifester sur tous les rejets d'une sous-unité de la souche et même parfois s'étendre à la quasi totalité de la cépée. L'application du marqueur, à l'automne, provoque des dégâts limités avant l'arrêt de la végétation. Le phytocide stocké durant la période hivernale est ensuite largement distribué au printemps suivant, dans toute la cépée pour le taillis d'un an, dans la sous-unité alimentée par la racine donneuse pour les taillis de 4 ans.

On met donc en évidence une voie de transport préférentielle en direction des rejets situés à proximité de la racine donneuse, mais les tissus de la souche jouent un rôle dans la distribution des substances apportées par les racines. La migration du marqueur paraît, d'autre part, beaucoup plus étendue dans le cas des cépées d'un an que dans celles de quatre ans.
\end{abstract}

Mots clés : Châtaignier, racine, souche, taillis, sectorisation.

\section{Introduction}

Les souches de châtaignier constituent des ensembles persistants qui garantissent la survie du taillis. Elles assurent des liens entre les rejets aériens et le système racinaire. Au cours des rotations successives, leur morphologie évolue par la formation de tissus nouveaux et la nécrose des parties anciennes. D'abord compactes, les souches se dissocient et des groupes de rejets, qui constituent des sous-unités, s'individualisent. Apparemment indépendantes, elles peuvent cependant être unies en profondeur par des tissus vivants. C'est sur les bases des tiges coupées ou « chicots " qu'apparaissent les nouveaux rejets. On peut se demander si les tissus du collet et de la souche jouent un rôle dans la répartition des éléments puisés dans le sol, s'ils assurent une distri- 
bution latérale des nutriments entre les divers rejets ou au contraire s'ils mettent en relation directe une racine ou un groupe de racines avec un rejet ou un groupe de rejets.

Chez le chêne-liège (Fagacées), Destremeau \& Roderiourg (1968) estiment que les rejets ont une vie autonome, la souche ne constituant qu'un support mécanique. Par contre, Bedeneau \& Pages (1984 b) ont montré que chez le bouleau (Bétulacées), les rejets d'une souche conservent pendant quelque temps des relations trophiques, l'individualisation des brins se faisant progressivement. Ainsi, deux espèces appartenant à des familles voisines semblent avoir des comportements différents. On peut donc se demander quel type de relation s'établit entre les racines et les rejets d'une souche de châtaignier, d'autant que Bedeneau \& Pagrs (1984 a) ont montré que des différences profondes se manifestent entre cette dernière essence et le bouleau, dans la croissance et le développement du système racinaire de la souche.

\section{Matériel et méthodes}

Nous avons utilisé des cépées dont les rejets ont 1 et 4 ans. L'âge des souches est inconnu. Elles sont constituées de sous-unités déjà individualisées, mais restent encore peu dissociées. Leur partie centrale est occupée par des tissus morts. Les restes des rejets de la rotation précédente constituent des chicols de diamètre variable. Ils avaient 20 ans au moment de l'abattage. Parmi les méthodes permettant d'étudier les relations entre les rejets et le système racinaire, certaines utilisent des éléments radioactifs (Destremau \& Roderhourg, 1968), d'autres des phytocides (Bedeneau \& Pages, 1984 b). Nous avons choisi d'employer un phytocide qui donne immédiatement une vue de l'ensemble des rejets atteints pour toute la cépée. Il permet en outre d'observer l'évolution, dans le temps, du mode de distribution.

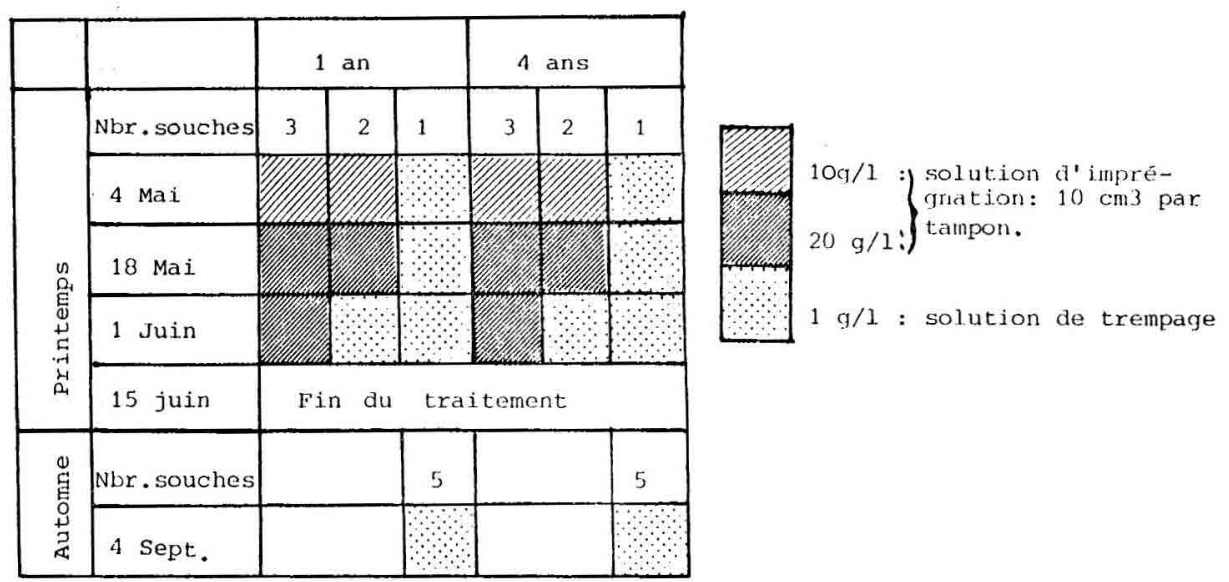

FIG. 1

Modalités d'application du phytocide sur les racines.

Levels of phytocid application on the roots. 
Cependant, une évaluation de la qualité de produit distribué est difficile et imprécise. Le phytocide utilisé ici est l'amitrole ( 3 amino 1,2, 4 triazole), transporté aussi bien par le phloème que par le xylène (CRAFTS, 1959 ; LEONARD et al., 1966). I1 provoque, en période de croissance active, un jaunissement très visible des feuilles, dont l'évolution et l'intensité permettent d'apprécier l'importance du transport. Deux séries d'expériences ont été réalisées : l'une au printemps (début : 4 mai 1984), l'autre en automne (début : 4 septembre 1984). Pour chaque classe d'âge, nous avons traité 6 cépées au printemps et 5 cépées en automne, soit un total de 22 cépées. Dans tous les cas, le marqueur a été appliqué sur une seule racine. Celle-ci a été choisie en fonction de sa position par rapport aux chicots : soit à la verticale de l'un d'eux, soit entre deux chicots unis à leur base par des tissus vivants. H s'agissait toujours de racines périphériques mesurant 2 à $3 \mathrm{~cm}$ de diamètre à $25 \mathrm{~cm}$ de la souche, point d'application du marqueur. Ces deux périodes de traitement, l'une au moment de la reprise de végétation, l'autre à la fin, ont été choisies de façon à déceler d'éventuelles modifications dans les relations racines-rejets, en fonction de la saison.
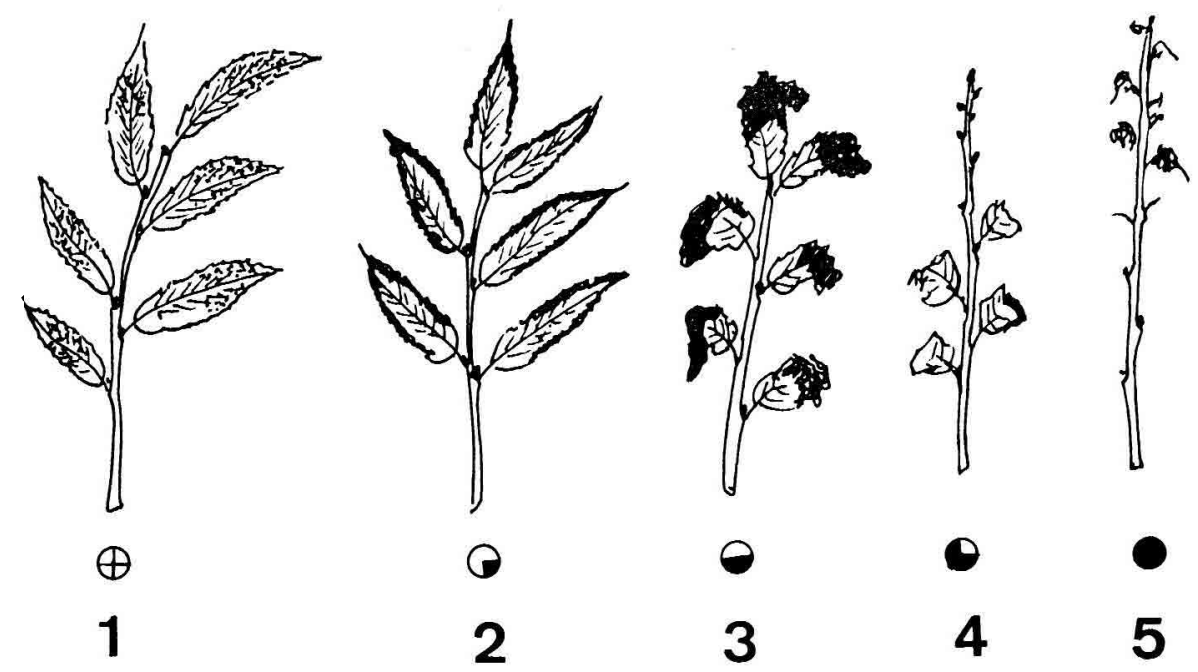

FIG. 2

Réaction des rejets au phytocide.

Reactions of shoots to phytocid application.

1. Traces de jaunissement aux extrémités des feuilles.

2. Marges des feuilles jaunes.

3. Marges et extrémités des feuilles brunies.

4. Chute des feuilles de la partie supérieure de la tige.

5. Rameau mort.

1. The tips of leaves turn yellow.

2. The edges of leaves turn yellow.

3. The edges and tips of leaves turn brown.

4. Fall of the leaves on the shoot-tips.

5. Dead shoot. 
Au printemps, pour suivre la progression de l'activité du phytocide, l'apport a été fait en plusieurs fois avec des concentrations croissantes (fig. 1). Sur cinq des six cépées traitées, on entoure d'un manchon de ouate de cellulose, une racine dont les tissus subérifiés sont grattés sur environ $5 \mathrm{~cm}$ de hauteur. Le tampon est imprégné de $10 \mathrm{ml}$ d'une solution d'amitrole à $10 \mathrm{~g} / 1$ et protégé par une feuille de plastique. L'apport est de $0,1 \mathrm{~g}$ de substance active. Quinze jours plus tard, l'opération est renouvelée avec une solution à $20 \mathrm{~g} / 1$. L'apport est de $0,2 \mathrm{~g}$. Quatre semaines après le début de l'expérience, sur deux souches, la racine traitée est sectionnée et plongée dans un sac en plastique contenant 3 litres d'une solution d'amitrole à $1 \mathrm{~g}_{j}^{\prime}$ ! L'apport est de $3 \mathrm{~g}$. Pour vérifier que ce dernier traitement ne modifie pas profon. dément le mode de distribution du phytocide, il est d'emblée utilisé sur la sixième souche. Les visites hebdomadaires des placettes d'expérience, ont permis de noter, pour chaque rejet, la date d'apparition des premiers effets du phytocide (tabl. 1 et 2). Au début de septembre, nous avons relevé l'intensité de la réaction et distingué cinq degrés selon la gravité des dégâts (fig. II).

TABLEAU 1

Souches portant des rejets d'un an:

nombre de rejets atteints et mode d'application du marqueur.

Stools with 1 year old shoots : number of affected shoots and method of phytocid application.

\begin{tabular}{|c|c|c|c|c|c|c|c|}
\hline $\begin{array}{l}N^{0} \text { de la } \\
\text { souche }\end{array}$ & 4 Mai & $18 \mathrm{Mai}$ & 25 Ma i & 1 Juin & 15 Juin & 29 Juin & 10 sept. \\
\hline 1 & $10 \mathrm{~g} / 1$ & ${ }^{20 g / 1} 7$ & 7 & 7 & 7 & 7 & 7 \\
\hline 2 & $10 \mathrm{~g} / 1$ & ${ }^{20 g / 1} 1$ & 1 & 1 & 3 & 6 & 7 \\
\hline 3 & $10_{\mathrm{g}} / 1$ & $20 \mathrm{gg} / 12$ & 2 & 2 & 2 & 5 & 7 \\
\hline 4 & $10 \mathrm{~g} / 1$ & $20 g / 11$ & 2 & $\begin{array}{l}\text { trempage } \\
\text { racine } \mathbf{2}\end{array}$ & 3 & 11 & 11 \\
\hline 5 & $10 \mathrm{~g} / 1$ & $20 \mathrm{~g} / 1$ & 2 & $\begin{array}{l}\text { trempage } \\
\text { racine } 2\end{array}$ & 2 & 15 & 15 \\
\hline 6 & $\begin{array}{l}\text { trempage } \\
\text { racine }\end{array}$ & 13 & 15 & 15 & 24 & 24 & 24 \\
\hline & 4 sept. & 18 Sept. & 2 oct. & & & & \\
\hline 7 & & 3 & 3 & & & & \\
\hline 8 & 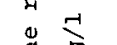 & 2 & 5 & & & & \\
\hline 9 & $\vec{r}$ & 9 & 9 & & & & \\
\hline 10 & 然 & 4 & 5 & & & & \\
\hline 11 & & 3 & 7 & & & & \\
\hline
\end{tabular}


A l'automne où la rapidité de la réponse a été recherchée, seule la méthode par section et immersion de la racine a été utilisée. Les observations ont été alors réalisées sur cinq cépées dans chaque classe d'âge (fig. I). Au cours des visites périodiques (tabl. 1), nous avons noté la date d'apparition des nécroses dues au phytocide mais le jaunissement naturel des feuilles en octobre ne nous a pas permis d'évaluer l'intensité de la réaction.

\section{Résultats}

\subsection{Expérience du printemps}

\subsection{Taillis d'un an}

Les jeunes rejets sont implantés sur les bases des chicots, à la périphérie de la souche et principalement dans le prolongement des racines.

- Le marqueur est appliqué par des tampons imprégnés d'amitrole (fig. III, $1,2,3)$. Les premières traces de jaunissement foliaire apparaissent deux semaines après l'application du phytocide. L'attaque est alors localisée aux rejets proches de la racine donneuse. L'augmentation de la concentration du phytocide ne produit que des effets lents et faibles. Après 6 à 8 semaines, le nombre de rejets atteints est peu important (tabl. 1). Situés latéralement par rapport à la racine traitée (fig. III, 2, 3), ils restent localisés dans le même secteur de la souche, sur la partie externe de celle-ci et à la base du même chicot. La présence de racines à proximité des rejets atteints, ne les empêche pas de recevoir le phytocide issu d'une racine plus éloignée. L'intensité de la réaction observée en fin d'expérience est maximale (degré 4) dans les premiers rejets atteints, proches de la racine donneuse. L'évolution de cette intensité pendant la durée de l'expérience n'a pas été notée car trop difficile à évaluer avec la méthode employée.

- Le marqueur est appliqué par des tampons durant 4 semaines, puis par trempage de la racine dans la solution (fig. III, 4,5). Le trempage de la racine déclenche après 3 ou 4 semaines un jaunissement des feuilles dans de nombreux rejets situés de part et d'autre de la racine donneuse. Les bases de deux chicots sont atteintes, un rejet situé au centre de la souche est lui-même faiblement touché : degré 1 . Ce transport latéral reste faible et lent.

- La racine est trempée directement dans la solution de phytocide (fig. III, 6). Le nombre des rejets atteints est important (tabl. 1). Au bout de deux semaines, 13 rejets appartenant à trois chicots différents sont jaunis. A l'aplomb de la racine donneuse, les rejets du centre de la souche sont eux aussi atteints. Au bout de trois semaines, une autre sous-unité est touchée bien qu'elle n'ait pas de relation visible avec la racine donneuse. Après six semaines, la moitié des rejets de la cépée sont atteints, un seul chicot est totalement épargné. Les rejets qui appartiennent à la sous-unité la plus proche de la racine donneuse sont les premiers et les plus fortement nécrosés.

- Au printemps suivant, nous n'avons pas noté de nouvelles traces de nécrose par rapport à l'automne. Les brins morts n'ont pas donné de rejets et les moins atteints (stades 1,2,3) ont repoussé. Quant à ceux moyennement atteints (stade 4), ils ont émis des feuilles totalement dépourvues de chlorophylle. 

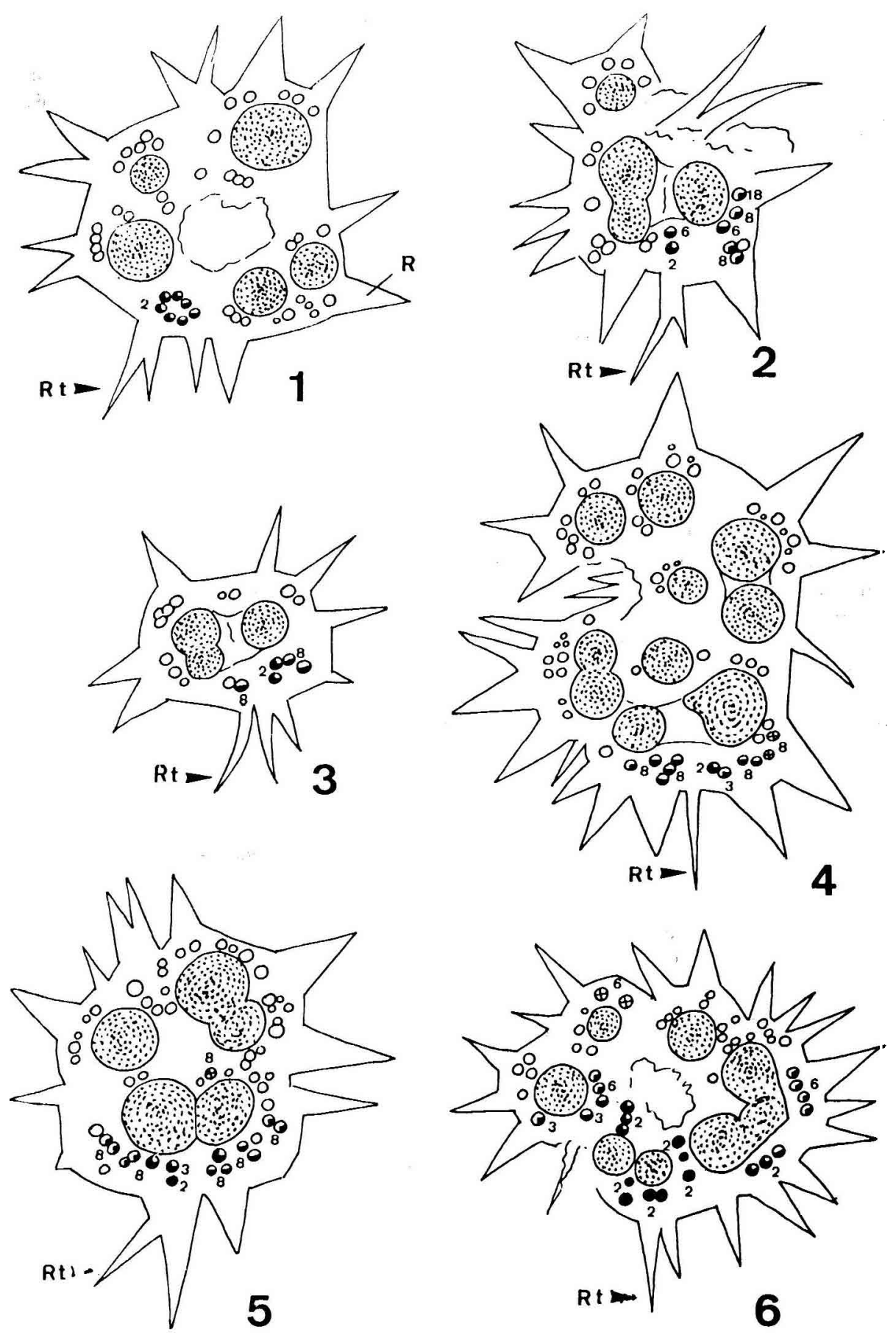


\subsection{Expérience d'automne}

Au bout de deux semaines, les rejets touchés sont encore peu nombreux. Ils peuvent appartenir au sous-ensemble de la racine donneuse (fig. IV, 7-10) ou même aux sous-unités voisines (fig. IV, 9-11) et être implantés aussi bien du côté interne que du côté externe des chicots. Au bout de quatre semaines, le nombre des rejets atteints augmente peu (fig. IV, 7, 8, 9, 10, 11).

Une visite effectuée lors du débourrement au printemps suivant montre que dans toutes les cépées traitées en septembre, le phytocide a atteint la quasi totalité des rejets. Ceux qui avaient été touchés en automne sont morts, les autres présentent des chloroses d'autant plus fortes qu'ils sont plus proches de la racine donneuse.

\subsection{Taillis de quatre ans}

Au printemps (fig. V, 12, 13, 14, 15, 16, 17), l'application de tampons imbibés de marqueur n'a provoqué qu'un faible jaunissement (degré 2 ou 1), apparu tardivement et toujours à proximité immédiate de la racine donneuse. Le trempage de la racine dans la solution augmente l'intensité de la réaction (degré 3) sans modification dans la répartition des rejets atteints, qui sont toujours disposés à la partie externe de la souche et sur les chicots de la sous-unité à laquelle appartient la racine donneuse. Les rejets atteints sont peu nombreux (tabl. 2).

A l'automne, aucune trace de jaunissement provoqué n'a pu être observée avant la chute des feuilles.

FIG. 3

Souches portant des rejets d'un an traités au printemps.

Stools with one year old shoots. Phytocid application is carried out in spring.

\subsubsection{Application de tampons imprégnés d'amitrole.}

4.5. Application de tampons durant quatre semaines, puis trempage de la racine dans la solution d'amitrole.

6. La racine est plongée dès le début dans la solution d'amitrole..

Rt : racine traitée.

L'importance des nécroses est figurée suivant le code de la fig. 2, 1, les chiffres indiquent le nombre de semaines séparant le début de l'expérience de l'apparition des nécroses.

Pour des raisons de clarté du dessin, nous avons volontairement grossi le diamètre des rejets par rapport à celui des chicots sans respecter l'échelle, nous n'avons retenu que la position relative des uns et des autres.

\subsubsection{Application of amitrol impregnated pads.}

4.5. Application of pads for 4 weeks, then, dipping the root into the amitrol solution.

6. The root was dipped into the amitrol solution from the beginning.

$R t$ : treated root.

The importance of damage is represented according to the code in fig. 2, 1. The numbers indicate the number of weeks between the beginning of the experiment and the appearance of the damage. 

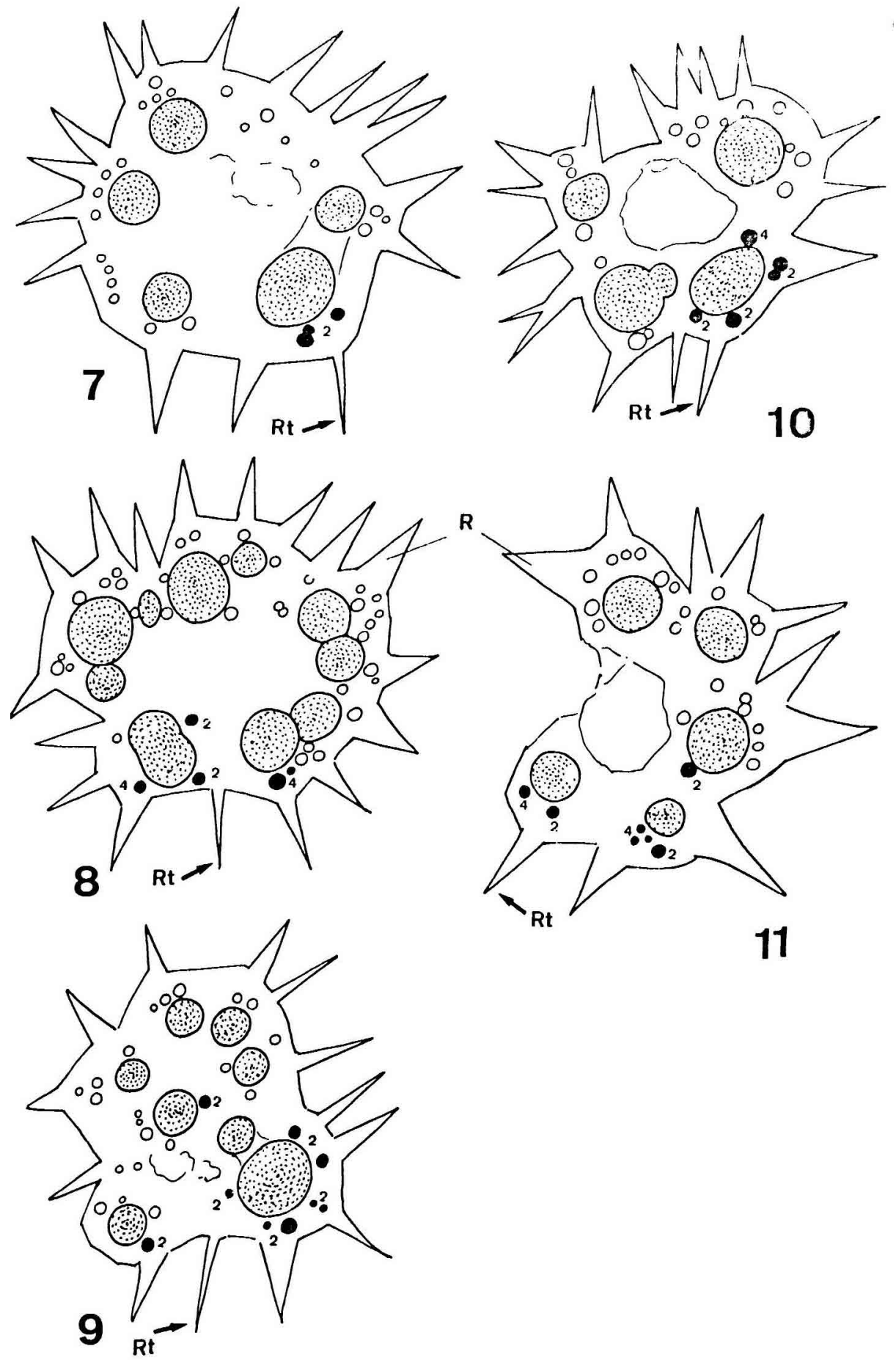


\section{TABleau 2}

Souches portant des rejets de 4 ans:

nombre de rejets atteints et mode d'application du marqueur.

Stools with 4 years old shoots : number of affected shoots and method of phytocid application.

\begin{tabular}{|c|c|c|c|c|c|c|c|}
\hline $\begin{array}{l}N^{0} \text { de la } \\
\text { souche }\end{array}$ & $4 \mathrm{Mai}$ & 18 Mai & $25 \mathrm{Mai}$ & 1 Juin & 15 Juin & 29 Juin & 10 sept. \\
\hline 12 & $10 g / I$ & $20 \mathrm{~g} / 1$ & 1 & 1 & 1 & 1 & 2 \\
\hline 13 & $10 \mathrm{~g} / 1$ & $20_{g} / 1$ & & 1 & 1 & 1 & 3 \\
\hline 14 & $10 \mathrm{~g} / 1$ & $20 \mathrm{~g} / 1$ & 1 & 1 & 1 & 1 & 1 \\
\hline 15 & $\log / 2$ & $20 \mathrm{~g} / 1$ & & $\begin{array}{r}\text { trempage } \\
\text { racinel }\end{array}$ & 3 & 3 & 4 \\
\hline 16 & $10 \mathrm{~g} / 1$ & $20 \mathrm{~g} / 11$ & 1 & $\begin{array}{r}\text { trempage } \\
\text { racine } 1\end{array}$ & 1 & 1 & 1 \\
\hline 17 & $\begin{array}{l}\text { Trempage } \\
\text { racine }\end{array}$ & & 5 & 5 & 5 & 5 & 6 \\
\hline
\end{tabular}

Comme pour le taillis d'un an, nous avons effectué une visite lors du débourrement au printemps suivant. Les rejets insérés à proximité immédiate de la racine donneuse sont morts. Chez quelques-uns, on peut cependant voir des bourgeons se développer sur leur base, et donner des feuilles chlorosées. Le phytocide peut atteindre, mais plus faiblement, les autres rejets de la sous-unité concernée.

\section{Discussion - Conclusion}

Les cépées se dissociant en sous-unités plus ou moins indépendantes, il est intéressant de considérer la distribution des substances tranportées par les racines au sein d'une sous-unité aussi bien que dans la cépée toute entière.

L'application de faibles quantités de phytocide met en évidence une voie de transport préférentielle vers les rejets les plus proches de la racine donneuse.

FIG. 4

Souches portant des rejets d'un an traités à l'automne.

Stools with one year old shoots. Phytocid application is carried out in autumn.

Dans cette figure, les rejets atteints sont noircis.

Legend: see fig. 3.

The blackened sprouts show damage occuring since autumn. 

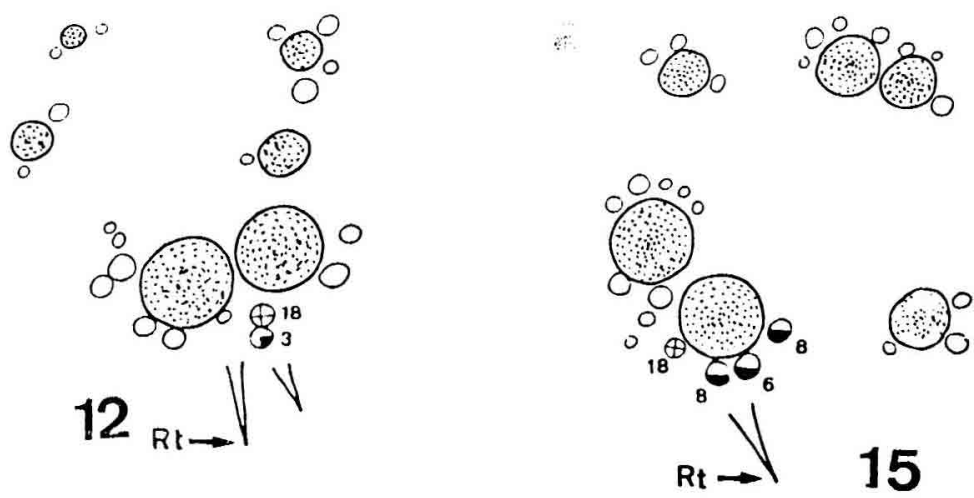

$0_{0}^{0} \bigcirc$
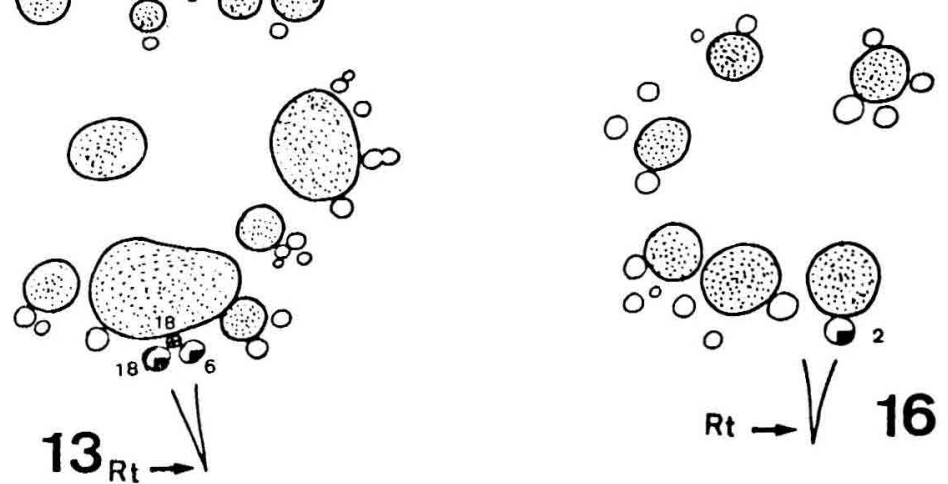

$R t \rightarrow / 16$
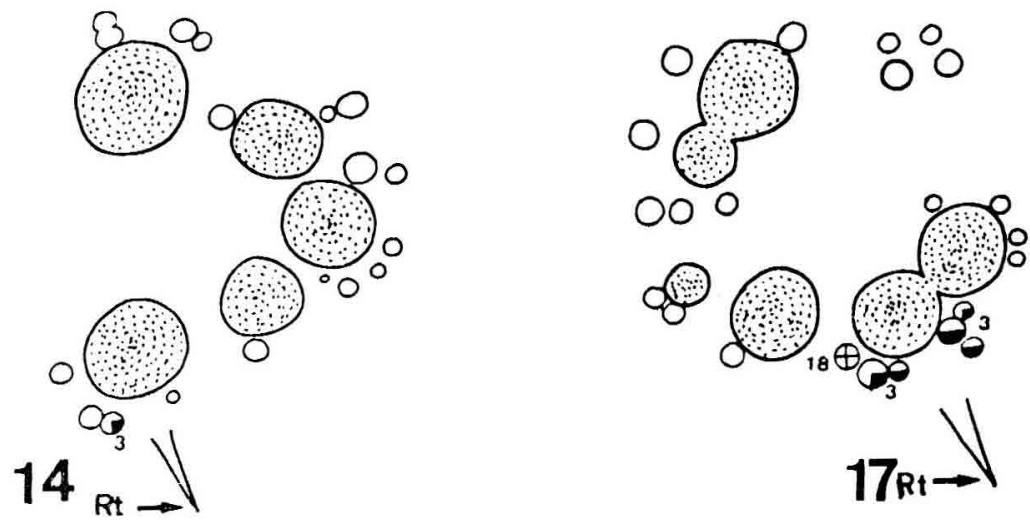

FIG. 5

Souches portant des rejets de quatre ans traités au printemps.

Stools with 4 years old shoots. Phytocid application is carried out in spring.

12.13.14. Application de tampons imprégnés d'amitrole.

15.16. Application de tampons durant quatre semaines, puis trempage de la racine dans la solution d'amitrole.

17. Racine trempée dès le début dans la solution d'amitrole.

12.13.14. Application of amitrol impregnated pads.

15.16. Application of impregnated pads for four weeks then dipping the root into the amitrol solution.

17. The root was dipped into the amitrol solution from the beginning. 
Les rejets situés au-dessus de la racine traitée sont les premiers atteinîs. Lorsque la quantité de phytocide transporté augmente, les dégâts s'étendent aux rejets latéraux de la sous-unité et plus tardivement à ceux qui s'insèrent sur lá face interne des chicots. Quelles que soient les modalités du traitement, les rejets les plus proches de la racine donneuse présentent donc toujours les premières et les plus graves atteintes. Le phytocide est ensuite distribué latéralement, d'autant plus vite que l'apport est plus important. Son effet s'atténue avec l'éloignement de la racine donneuse.

Cependant, les relations privilégiées existant entre une racine et les rejets les plus proches n'excluent pas la possibilité d'apports à partir d'autres racines. En effet, même lorsque la réaction au phytocide est peu étendue, certains des rejets atteints sont plus proches d'une racine intacte que de la racine donneuse. En cas d'apport plus important, le marqueur peut atteindre des rejets très éloignés de la racine donneuse (souche $n^{\circ} 6$, fig. III).

Durant la période hivernale, on assiste à un stockage du phytocide absorbé à l'automne. Il sera très largement distribué au printemps suivant : dans toute la cépée pour le taillis d'un an, dans la sous-unité concernée pour le taillis de quatre ans.

De toute évidence, les tissus de la souche jouent un rôle dans la répartition des substances apportées par les racines, entre les différents rejets et on ne peut pas, comme l'ont fait Destremeau \& Roderbourg, pour le chêne-liège, considérer la souche comme un simple support auquel aboutissent des racines qui alimentent un rejet particulier. Comme l'ont montré Bedeneau \& PAges chez le bouleau, on peuí mettre en évidence, dans la cépée de châtaignier des relations privilégiées qui s'établissent, suivant un critère de proximité, entre une racine et quelques rejets.

Cependant, les tissus de la souche permettent un transport lent vers les autres rejets appartenant à la sous-unité alimentée par la racine donneuse. Un transport à plus grande distance a pu être également observé. Il révèle alors des relations complexes qui n'obéissent plus au seul critère de proximité. Ce phénomène a été également mis en évidence chez le bouleau.

On note, d'autre part, des modifications du modèle de distribution du traceur en fonction de l'âge des rejets de la cépée, sa répartition étant généralement beaucoup plus étendue lorsque les pousses ont un an que lorsqu'elles ont quatre ans. La dilution plus importante du phytocide en raison de l'augmentation du volume des brins paraît insuffisante pour expliquer le phénomène. On doit certainement envisager une modification des voies de transport existant dans la souche à la suite de la destruction progressive des anciens chicots, la formation de tissus jeunes et, comme l'ont montré Bedeneau \& PAGEs, la production de nouvelles racines. Cette évolution permettrait d'envisager à plus long terme, dans du taillis âgé, un modèle de distribution des substances apportées par les racines, plus proche de celui observé par Destremeau \& Roderbourg chez le chêne-liège. En effet, les expérimentations menées sur cette essence ont été faites sur des cépées portant seulement 3 à 4 rejets âgés de 10 ans. Il est possible que, dans ces conditions, chaque brin ait alors acquis une certaine indépendance.

Si les tissus de la souche peuvent donc participer à la distribution plus ou moins étendue des substances transportées par une racine, on peut se demander dans quelle mesure la suppression des rejets est capable de modifier les voies de transport établies. Il serait, à ce sujet, intéressant d'étudier le trajet des substances transportées par une racine après l'élimination des rejets prioritairement 
alimentés. Les expériences réalisées ici nous placent partiellement dans cette situation : l'application de fortes quantités de phytocide tuant rapidement les rejets situés à proximité de la racine donneuse.

\author{
Reçule 2 juin 1985. \\ Accepté le 23 décembre 1985.
}

\title{
Remerciements
}

Cette étude s'est déroulée dans le cadre d'une convention passée entre l'Université de limoges et le C.E.M.A.G.R.E.F. de Nogent-sur-Vernisson. Nous tenons à remercier cet organisme pour laaide qui nous a été ainsi apportée. Nos remerciements vont aussi à M"' Gérardin qui nous a autorisé à expérimenter dans sa propriété.

\section{Summary}

\section{The relationship between a root and Castanea stool shoots}

The stools of coppiced Castanea are the perennial part of the tree which maintains the link between the shoots and the root system. The new shoots appear on the remnants of the cut stems. We wondered whether the stool-tissue is involved in the diffusion of nutrient elements drawn from the soil, and whether it is responsible for lateral transfer, or conversely, whether there is a direct relationship between a root and a shoot. The results obtained by Destrfmau and Rodebouri; (1968) with Quercus suber were very different from those of Bedeneau and Pasies (1984 b) with Betula. Here we propose to study what happens with a similar variety : Castanea.

\section{Method}

A phytocid (amitrol) was applied to one root of different Castanea stools. The experiment was carried out on one and four year old bushes. In both age-categories, 6 clumps of shoots were treated in the spring, and 5 in the autumn. The amitrol application was made in several stages with ever increasing concentrations. Visits at regular intervals enabled us to note when the first damage appeard in each shoot. At the end of the experiment, we note how strong the reaction was, and identified 5 levels according to whether the damage as more or less serious. The necrosis ranged from yellowish tinges on the leaves, to the death of the shoot.

\section{Results}

During the spring experiment on the one year old bushes, the first results appeard two weeks after the beginning of the treatment. When the dose of phytocid was weak, the damaged shoots were to be found near the treated root : the leaves turned yellow. As the amount of phytocid in the plant increased, the damage spread to the lateral shoots. The further the root was away, the weaker the reaction. The results were similar for the autumn experiment. After a visit made the following spring, we concluded that the phytocid stored during the winter period, was largery distributed in the spring. The damage is always more serious on the stools with young shoots.

\section{Conclusion}

The use of small quantities of phytocid reveals a method of preferential diffusion, but the stool-tissue may also play a role in the storage and the widespread diffusion of the absorbed product. As far as sweet chestnut and birch are concerned (BEDENenu \& PAGEs, 1984), the stool does not merely act as a support, it also plays an active part in the diffusion of substances transported by the roots.

Key words : Chestnut sweet, root, stool, coppice, segmentation. 


\section{Références bibliographiques}

Bedeneau M., Pages L., 1984 a. Etude des cernes d'accroissement ligneux du système racinaire d'arbres traités en taillis. Ann. Sci. For., 41 (1), 59-68.

Bedeneau M., Pages L., 1984 b. Répartition de la sève entre les jeunes rejets de bouleau étudiés à l'aide d'un phytocide. Ann. Sci. For., 41 (2), 131-141.

Crafts A.S., 1959. Further studies on comparative mobility of labelled herbicides. Plant Physiol., 34, 613-620.

Destremau D.X., Roderbourg J., 1968. Eléments pour l'étude du traitement en taillis de la forêt de chêne-liège de Mamora. Répartition de la sève entre les rejets. $A n n$. Rech. For. au Maroc, 2, rapport 1968-1969, 237-242.

Leonard O.A., Lider L.A., GlenN R.K., 1966. Absorption and translocation of herbicides by Thompson seedless (Sultanina) Grape, Vitis vinifera L., Weed Res., 6, 37-49. 